\title{
Impacto de los inhibidores de la bomba de protones en los niveles de vitamina B12 en pacientes con ERC estadio 5 en hemodiálisis Experiencia de un centro en Manizales, Colombia
}

\author{
Impact of proton-pump inhibitors on vitamin B12 \\ levels in patients with CKD stage 5 in hemodialysis \\ Experience of a Center in Manizales, Colombia
}

\author{
Carlos Hernán Restrepo, John Jairo Botello, Lina María López, \\ José Vicente Aguirre, César Augusto Restrepo • Manizales (Colombia)
}

\section{Resumen}

Objetivo: establecer si en pacientes con enfermedad renal crónica (ERC) estadio 5 en terapia de hemodiálisis y uso crónico de inhibidores de bomba de protones (IBPs) se presenta deficiencia de vitamina B12.

Métodos: pacientes con ERC estadio 5 mayores de 18 años, en terapia de hemodiálisis crónica, quienes se encontraban recibiendo terapia con IBP. En ellos se evaluaron variables demográficas, antropométricas, etiología de la enfermedad renal crónica, tiempo en diálisis, tipo de IBP que el paciente estaba recibiendo, dosis y tiempo que llevaban usando el medicamento. Las variables de laboratorio relacionadas fueron concentraciones séricas de vitamina B12, ácido fólico, KTV, hemoglobina y volumen corpuscular medio

Tipo de estudio: analítico de corte transversal. Para el análisis estadístico se utilizaron medidas de tendencia central y de dispersión (media, desviación estándar) en las variables cuantitativas y frecuencias relativas en las cualitativas. Para determinar la correlación entre los niveles de vitamina B12 y las variables se utilizó el método de regresión lineal simple para cada una de las variables continuas y el de regresión logística para las categóricas

Resultados: de un total de 106 pacientes en terapia de hemodialisis crónica, 37 pacientes cumplieron con los criterios de inclusión, 45.9\% mujeres, edad promedio 63.6 años, etiología de la ERC $48.6 \%$ idiopática. Respecto al tiempo en diálisis $56.7 \%$ de los pacientes tenían menos de cuatro años, el resto entre cuatro y 19 años. En cuanto al tiempo de consumo de IBP 15 pacientes (40.5\%) habían recibido al menos tres años y 22 pacientes $(59.5 \%)$ más de tres años. El 100\% de los pacientes exhibían cifras normales o por encima de lo normal de niveles séricos de ácido fólico. Se encontró correlación positiva, aunque no estadísticamente significativa entre los niveles séricos de vitamina B12 y la dosis de IBP, tiempo de consumo de IBP y tiempo en diálisis.

Conclusiones: contrariamente a lo planteado en la hipótesis de trabajo, en este estudio se encontró que los valores séricos de vitamina B12, tienden a aumentar a mayor tiempo en diálisis y el consumo de IBP, tanto en tiempo, como en dosis (Acta Med Colomb 2017; 42: 172-179).

Palabras clave: diálisis renal, enfermedad renal crónica inhibidores de la bomba de protones, vitamina B12.

\footnotetext{
Abstract

Objective: To determine if vitamin B12 deficiency occurs in patients with chronic renal disease (CKD) stage 5 in hemodialysis therapy and chronic use of proton pump inhibitors (PPIs).

Methods: patients with stage 5 CKD over 18 years of age on chronic hemodialysis therapy who were receiving PPI therapy. Demographic and anthropometric variables, chronic renal disease etiology, time on dialysis, type of PPI received by the patient, dose and time taking the medication, were evaluated. The related laboratory variables were serum levels of vitamin B12, folic acid, KTV, hemoglobin and mean corpuscular volume.
}

Carlos Hernán Restrepo Duque: Estudiante de Medicina; Dr. John Jairo Botello Jaimes: Magister en Educación, Doctorando en Educación, Director del Programa de Medicina, Docente de Medicina Interna; Dra. Lina María López Castro: Médica General, Médico de Apoyo RTS; Dr. José Vicente Aguirre Arango: Salubrista Epidemiólogo Mg en Salud Publica, Profesor Catedrático. Universidad de Manizales; Dr. César Augusto Restrepo Valencia: Internista Nefrólogo. Profesor Asociado Universidad de Caldas y Universidad de Manizales. Manizales (Colombia).

Correspondencia. Dr. Cesar Augusto Restrepo Valencia. Manizales (Colombia).

E-mail: caugustorv@une.net.co, cesar.restrepo_v@ucaldas.edu.co

Recibido: 16/VII/2016 Aceptado: 19/V/2017 
Type of Study: cross-sectional analytical. Statistical analysis used central tendency and dispersion measures (mean, standard deviation) in the quantitative variables and relative frequencies in the qualitative ones. To determine the correlation between vitamin B12 levels and variables, the simple linear regression method was used for each of the continuous variables and the logistic regression method for the categorical variables.

Results: Of a total of 106 patients undergoing chronic hemodialysis therapy, 37 patients met the inclusion criteria. $45.9 \%$ were women, mean age 63.6 years, etiology of CKD $48.6 \%$ idiopathic. Regarding the time on dialysis $56.7 \%$ of the patients had less than four years; the rest between four and 19 years. Regarding the time of PPIs consumption, 15 patients $(40.5 \%)$ had received at least three years and 22 patients $(59.5 \%)$ more than three years. $100 \%$ of the patients exhibited normal or above normal levels of serum folic acid levels. A positive correlation was found, although not statistically significant, between serum vitamin B12 levels and PPI dose, PPI consumption time and dialysis time.

Conclusions: Contrary to what was proposed in the hypothesis of this study, it was found that serum levels of vitamin B12 tend to increase over time in dialysis and PPI consumption, both in time and in doses (Acta Med Colomb 2017; 42: 172-179).

Keywords: renal dialysis, chronic kidney disease, proton pump inhibitors, vitamin B12.

\section{Introducción}

La vitamina B12 es esencial para el correcto funcionamiento del cuerpo humano, tanto en su desarrollo como en el sostenimiento de múltiples funciones biológicas en la edad adulta. No es producida endógenamente, por lo tanto debe ser absorbida en la dieta y se encuentra fundamentalmente en las proteínas animales. Su déficit produce complicaciones graves como anemia megaloblástica, deterioro neurológico y demencia. El organismo tiene efectivos sistemas de absorción y almacenamiento de dicha vitamina, y se requiere un periodo prolongado de escasez para generar las manifestaciones clínicas asociadas a su déficit (1).

Los pacientes con enfermedad renal crónica podrían ser especialmente vulnerables a desarrollar deficiencia de dicha vitamina, puesto que requieren con frecuencia dietas hipoproteicas para el control de la hiperfosfatemia e hiperpotasemia. Para los pacientes en hemodiálisis las pérdidas de vitaminas hidrosolubles filtradas en la terapia dialítica pueden contribuir a acrecentar el déficit (2).

El uso de inhibidores de la bomba de protones podría disminuir la absorción de la vitamina B12, pues ésta es dependiente del medio ácido estomacal que se encarga de separarla de las proteínas ingeridas en los alimentos (3). Los pacientes con enfermedad renal crónica con frecuencia utilizan inhibidores de la bomba de protones (IBPs) para el control de la gastritis urémica, y con el fin de modular el PH gástrico que se puede afectar por enfermedades y medicamentos que tienden a acidificarlo (4).

El propósito de esta investigación, fue analizar el impacto que podría tener el tiempo de exposición a los IBPs en los niveles séricos de vitamina B12 en pacientes en hemodiálisis crónica en una unidad renal en la ciudad de Manizales/ Colombia. Se esperaba determinar si a mayor dosis y tiempo de exposición de los IBPs existía una mayor prevalencia de déficit de vitamina B12.

\section{Material y métodos}

Área de estudio: unidad renal de RTS sucursal Caldas, en Manizales, departamento de Caldas, Colombia.

Se evaluaron 106 pacientes mayores de 18 años, cualquier género y raza residentes en Manizales, con diagnóstico de enfermedad renal crónica (ERC) estadio 5 en programa de hemodiálisis crónica, cubiertos por el plan obligatorio de salud, en quienes se interrogó y revisó las historias clínicas, en relación al tratamiento con inhibidores de la bomba de protones y el tiempo por el cual lo habían consumido

Los criterios de exclusión fueron: KT/V menor a 1.2, alcoholismo, fumador activo, gastritis atrófica confirmada por endoscopia digestiva alta, gastrectomía parcial o total, consumo de medicamentos que alteraran la absorción de vitamina B12 (metformina, fenobarbital, neomicina, colchicina y colestiramina), síndrome de malabsorción, déficit de ácido fólico, y terapia activa con vitamina B12.

Se registraron variables demográficas, antropométricas y clínicas: género, edad, grupo étnico, peso, talla, índice de masa corporal (IMC), etiología de la enfermedad renal crónica y tiempo en diálisis.

El índice de masa corporal (IMC) se obtuvo por aplicar la fórmula:

$\mathrm{IMC}=$ peso (en kilogramos) / altura $\left(\mathrm{en}^{2}\right.$ ), y se definió así: bajo peso menor a 18.49, Normal 18.5 a 24.99, sobrepeso 25 a 29.99 , obeso mayor de 30.

Las variables de laboratorio recolectadas fueron: concentraciones séricas de vitamina B12 y ácido fólico, KTV, hemoglobina y volumen corpuscular medio (VCM). Se anotó el tipo de IBP que el paciente estaba recibiendo, su dosis y el tiempo de uso del medicamento.

Los valores diagnósticos para deficiencia de vitamina B12 fueron: valor sérico de vitamina B12 menor a $200 \mathrm{Pg} / \mathrm{mL}$. Los valores séricos entre 200 y $299 \mathrm{Pg} / \mathrm{mL}$ fueron considerados déficit marginal y los valores por en- 
cima de $300 \mathrm{Pg} / \mathrm{mL}$ normales (5). Los valores de ácido fólico debieron estarnormales para todos los pacientes $(2.7-17 \mathrm{ng} / \mathrm{mL})$, descartando la presencia de anemia megaloblástica debida a su déficit. Para el volumen corpuscular se definieron valores normales entre 80 y 96 fentolitros, y para la hemoglobina entre 10 y $11.5 \mathrm{~g} / \mathrm{dL}$.

Se consideraron valores adecuados para el KTV igual o por encima de 1.2, los cuales deberían estar presentes en todos los pacientes, reflejando que se encontraban recibiendo una diálisis efectiva, puesto que el KTV evalúa la eficiencia del procedimiento dialítico en su capacidad de remoción de la urea.

Se anotaron los medicamentos que recibía el paciente, y los que podrían tener relación con una alteración en los valores de vitamina B12.

El proyecto fue aprobado por el Comité de Bioética de la Universidad de Caldas, y Comités de Ética e Investigación de RTS (Servicio de Terapia Renal) para Colombia.

\section{Tipo de estudio}

Analítico de corte transversal prospectivo.

Aspectos éticos: de acuerdo con la Resolución 8430 de 1993 del Ministerio de Salud de Colombia, el estudio se clasificó en la categoría de Investigación de Riesgo Mínimo.

\section{Análisis estadístico}

Para la digitación de la información se diseñó una base de datos en Excel, la cual fue diligenciada por los investigadores. Para el procesamiento de la información y el cálculo de las pruebas estadísticas, se utilizó el paquete estadístico SPSS 23.0.

Para el cálculo del tamaño de muestra, y para estimar la correlación entre el uso crónico de inhibidores de bomba de protones en pacientes con ERC estadio 5 en terapia de

Tabla 1. Descripción de la población de acuerdo a las características demográficas ( $n=37)$.

\begin{tabular}{|l|c|c|c|c|c|c|}
\hline \multirow{2}{*}{$\begin{array}{c}\text { Grupos de edad } \\
\text { (años) }\end{array}$} & \multicolumn{2}{|c|}{ Mujeres } & \multicolumn{2}{c|}{ Hombres } & \multicolumn{2}{c|}{ Total } \\
\cline { 2 - 7 } & No. & $\%$ & No. & $\%$ & No. & $\%$ \\
\hline Menores de 30 & 1 & 100.0 & 0 & 0.0 & 1 & 100.0 \\
\hline De 30 a 39 & 2 & 66.7 & 1 & 33.3 & 3 & 100.0 \\
\hline De 40 a 49 & 2 & 50.0 & 2 & 50.0 & 4 & 100.0 \\
\hline De 50 a 59 & 2 & 33.3 & 4 & 66.7 & 6 & 100.0 \\
\hline De 60 a 69 & 2 & 50.0 & 2 & 50.0 & 4 & 100.0 \\
\hline De 70 a 79 & 6 & 50.0 & 6 & 50.0 & 12 & 100.0 \\
\hline De 80 a 89 & 2 & 28.6 & 5 & 71.4 & 7 & 100.0 \\
\hline Total & $\mathbf{1 7}$ & $\mathbf{4 5 . 9}$ & $\mathbf{2 0}$ & $\mathbf{5 4 . 1}$ & $\mathbf{3 7}$ & $\mathbf{1 0 0 . 0}$ \\
\hline
\end{tabular}

hemodiálisis y la deficiencia de vitamina B12 se tuvieron en cuenta parámetros como una confianza $\left(\mathrm{z}_{1-\alpha / 2}\right)$ del $95.0 \%$, un poder $\left(\mathrm{z}_{1-\beta}\right)$ del $80 \%$ y un coeficiente de correlación de Pearson (r) de 0.45 .

La población considerada UNIVERSO en este caso fue de 106 pacientes, y el número que se proyectó analizar de esta población fue 36 .

Para el análisis estadístico se utilizaron medidas de tendencia central y de dispersión (media, desviación estándar) en las variables cuantitativas y frecuencias relativas en las cualitativas. Para determinar la correlación entre los niveles de vitamina B12 y las variables: tiempo en diálisis, tiempo de consumo de IBPs, dosis de IBP, VCM y hemoglobina se utilizó el método de regresión lineal simple para cada una de las variables continuas y el de regresión logística para las categóricas.

\section{Resultados}

En RTS sucursal Caldas en el momento de la recolección de datos se encontraron 106 pacientes en terapia de hemodiálisis crónica. Después de aplicar los criterios de exclusión se logró obtener una muestra de 37 pacientes que cumplieron con los criterios de inclusión. De éstos, el $45,9 \%$ eran mujeres. El promedio de edad fue de 63.6 años, observándose un alto porcentaje de las personas con edades entre 70 y 79 años de edad (Tabla 1).

Con respecto a la etiología de la ERC se encontró que un poco menos de $50 \%$ era idiopática, le continuaba la nefropatía diabética en el $29.7 \%$. El resto de etiologías se presentan con frecuencias muy bajas (Tabla 2).

$\mathrm{Al}$ analizar el tiempo en la terapia en hemodiálisis se encontró que el 56,7\% de las personas valoradas, tenían en ella menos de cuatro años, el resto se distribuyeron entre 4 y 19 años (Tabla 3).

Tabla 2. Etiología de la ERC.

\begin{tabular}{|l|c|c|}
\hline \multicolumn{1}{|c|}{ Etiología ERC } & No. & $\%$ \\
\hline Idiopática & 18 & 48,6 \\
\hline Nefropatía diabética & 11 & 29,7 \\
\hline Hipertensión arterial y diabetes mellitus & 2 & 5,4 \\
\hline Nefropatía obstructiva & 2 & 5,4 \\
\hline Nefropatía lúpica & 1 & 2,7 \\
\hline GMN RP & 1 & 2,7 \\
\hline Nefropatía hipertensiva & 1 & 2,7 \\
\hline ERPQAD & 1 & 2,7 \\
\hline Total & $\mathbf{3 7}$ & $\mathbf{1 0 0 , 0}$ \\
\hline $\begin{array}{l}\text { GMN RP (glomerulonefritis rápidamente progresiva). } \\
\text { ERPQAD (enfermedad renal poliquística autosómica dominante). }\end{array}$ & \\
\hline
\end{tabular}


Para el tiempo en consumo de IBPs se clasificaron los pacientes en dos grupos: el grupo uno aquellos con menos o igual a tres años, encontrando15 pacientes (40.5\%), y para el grupo dos, más de tres años, 22 pacientes (59.5\%), esto demuestra que la prescripción crónica de IBPs es muy común en pacientes con ERC (Tabla 4).

$\mathrm{Al}$ caracterizar las variables de interés (edad, peso, talla, IMC, niveles vitamina B12, ácido fólico, KTV, VCM, tiempo de diálisis y tiempo de IBP) y compararlas por género, se encontró que el promedio en la mayoría es superior en los hombres, excepto en el IMC, KTV y VCM; sin embargo la desviación estándar de un alto porcentaje de las mediciones, es superior en las mujeres, indicando esto que hay más variabilidad en los resultados de ellas, que en la de los hombres.

En el análisis estadístico por género, sólo se encontraron diferencias estadísticamente significativas entre los promedios en la talla, KTV y VCM (Tabla 5).

Los resultados de los valores de ácido fólico analizados fueron normales o por encima de lo normal en $100 \%$ de los pacientes. El $75.7 \%$ de los pacientes tenía el VCM dentro de rangos normales, y en $21.6 \%$ por arriba de lo normal. Los niveles de hemoglobina fueron bajos en $10.8 \%$ de los pacientes (Tabla 6).

Se encontró correlación positiva (aunque no estadísticamente significativa) entre los niveles séricos de vitamina B12 y la dosis de IBPs, el tiempo de consumo de IBPs y el tiempo en diálisis. Lo anterior indica que contrariamente a lo planteado en la hipótesis de trabajo los valores séricos de vitamina B12, tienden a aumentar a medida que aumenta el tiempo en diálisis y el consumo de IBPs, tanto en tiempo, como en dosis (Tabla 7, Figuras 1,2)
Tabla 3. Tiempo en terapia de hemodiálisis.

\begin{tabular}{|l|c|c|}
\hline \multirow{2}{*}{ Tiempo diálisis en años } & \multicolumn{2}{|c|}{ Total } \\
\cline { 2 - 3 } & No. & $\%$ \\
\hline Menores de un año & 10 & 27.0 \\
\hline De 1-4 & 11 & 29.7 \\
\hline De 5-9 & 8 & 21.6 \\
\hline De 10-14 & 5 & 13.5 \\
\hline De 15-19 & 3 & 8.1 \\
\hline Total & $\mathbf{3 7}$ & $\mathbf{1 0 0 . 0}$ \\
\hline
\end{tabular}

Tabla 4. Tiempo de consumo de IBP.

\begin{tabular}{|l|c|c|}
\hline \multirow{2}{*}{ Tiempo IBP en años } & \multicolumn{2}{|c|}{ Total } \\
\cline { 2 - 3 } & No. & $\%$ \\
\hline Menor o igual a 3 & 15 & 40.5 \\
\hline Mayor a 3 & 22 & 59.5 \\
\hline Total & $\mathbf{3 7}$ & $\mathbf{1 0 0 . 0}$ \\
\hline
\end{tabular}

Resultados similares a los anteriores, se presentaron al considerar una variable dependiente (Niveles de vitamina $\mathrm{B} 12, \mathrm{VCM}, \mathrm{Hb}$ ) con dos independientes (tiempo IBP y tiempo de diálisis); para ninguno de los casos resultó un coeficiente de correlación superior a 0.40 , demostrando esto, que hay una correlación muy débil entre estas variables. Se apoya esta conclusión, con los valores p de las variables (Tabla 8).

Tabla 5. Análisis estadístico de diferencias entre variables por género.

\begin{tabular}{|c|c|c|c|c|c|c|}
\hline \multirow{2}{*}{ Variables } & \multicolumn{2}{|c|}{ Mujeres (n=17) } & \multicolumn{2}{|c|}{ Hombres (n=20) } & \multirow{2}{*}{ Valor $\mathbf{F}$} & \multirow{2}{*}{ Valor $\mathbf{p}$} \\
\hline & Promedio & DE & Promedio & DE & & \\
\hline Edad & 60.2 & 18.2 & 66.4 & 13.8 & 1.39 & 0.2461 \\
\hline Peso & 59.0 & 12.8 & 63.3 & 11.7 & 1.14 & 0.2925 \\
\hline Talla & 1.6 & 0.1 & 1.7 & 0.1 & 12.8 & 0.00094 \\
\hline IMC & 23.9 & 4.3 & 22.8 & 2.6 & 0.92 & 0.3463 \\
\hline Niveles Vitamina B12 & 623.8 & 215.4 & 750.0 & 287.7 & 2.21 & 0.1449 \\
\hline Ácido Fólico & 21.6 & 25.4 & 25.3 & 26.1 & 0.19 & 0.6659 \\
\hline KTV & 1.53 & 0.28 & 1.36 & 0.2 & 4.61 & 0.0380 \\
\hline $\mathrm{VCM}$ & 94.6 & 6.8 & 88.7 & 6.5 & 7.26 & 0.0103 \\
\hline Tiempo diálisis (años) & 3.9 & 3.4 & 7.22 & 6.2 & 2.06 & 0.1617 \\
\hline Tiempo IBP (años) & 3.3 & 2.6 & 4.2 & 3.6 & 0.74 & 0.3965 \\
\hline Hemoglobina & 11.4 & 1.6 & 12.4 & 0.97 & 2.24 & 0.1469 \\
\hline
\end{tabular}


Tabla 6. Resultados de los valores de ácido fólico, VCM y hemoglobina.

\begin{tabular}{|l|c|c|c|c|c|c|}
\hline \multirow{2}{*}{ Valoración } & \multicolumn{2}{|c|}{ Ácido fólico } & \multicolumn{2}{c|}{$\begin{array}{c}\text { Volumen } \\
\text { corpuscular }\end{array}$} & \multicolumn{2}{c|}{$\begin{array}{c}\text { Hemoglobina } \\
\text { (Hb) }\end{array}$} \\
\cline { 2 - 7 } & No. & $\%$ & No. & $\%$ & No. & $\%$ \\
\hline Normal & 21 & 56.8 & 28 & 75.7 & 12 & 32.4 \\
\hline Por encima de lo normal & 16 & 43.2 & 8 & 21.6 & 21 & 56.8 \\
\hline Por debajo de lo normal & 0 & 0.0 & 1 & 2.7 & 4 & 10.8 \\
\hline Total & $\mathbf{3 7}$ & $\mathbf{1 0 0 . 0}$ & $\mathbf{3 7}$ & $\mathbf{1 0 0 . 0}$ & $\mathbf{3 7}$ & $\mathbf{1 0 0 . 0}$ \\
\hline
\end{tabular}

Tabla 7. Correlación entre niveles séricos de vitamina B12 y las variables tiempo en diálisis e IBP.

\begin{tabular}{|l|c|c|c|}
\hline Variables & Correlación & Valor p & $\mathbf{n}$ \\
\hline Niveles vitamina B12 - dosis IBP & 0.169 & 0.318 & 37 \\
\hline Niveles vitamina B12 - tiempo IBP & 0.008 & 0.963 & 37 \\
\hline Niveles vitamina B12 - tiempo diálisis & 0.111 & 0.513 & 37 \\
\hline Tiempo IBP - VCM & -0.127 & 0.454 & 37 \\
\hline Tiempo diálisis - VCM & -0.108 & 0.526 & 37 \\
\hline
\end{tabular}

Se dividieron además los pacientes en grupos de tiempo de consumo de IBP, con el fin de evaluar si los pacientes con mayor tiempo en su consumo presentaban más posibilidades de detectarse niveles bajos de vitamina B12. Las Figuras 3, 4 y 5 demuestran que no hubo diferencias importantes en los resultados.

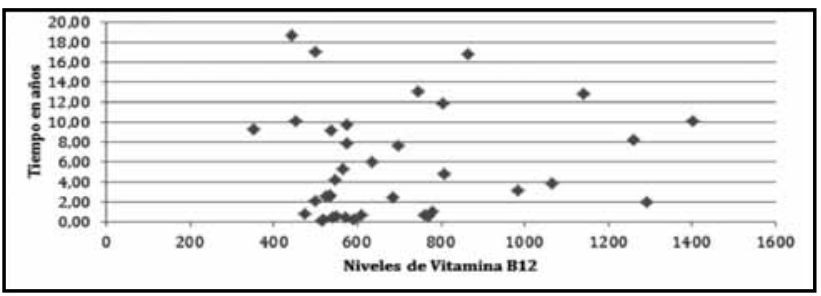

Figura 1. Relación entre niveles de vitamina B12 y tiempo en hemodiálisis.

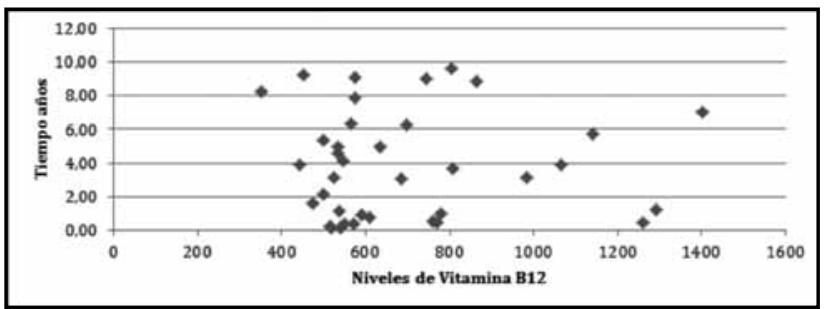

Figura 2. Relación entre niveles de vitamina B12 y uso de IBP en el tiempo.

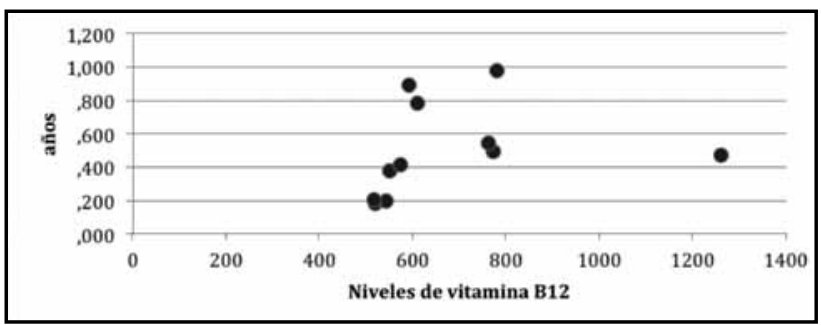

Figura 3. Relación entre niveles de vitamina B12 y uso de IBP por menos de un año.

Tabla 8. Correlación entre niveles séricos de vitamina B12 y las variables tiempo en diálisis e IBP. Consideración de una variable dependiente con dos independientes.

\begin{tabular}{|c|c|c|c|c|c|}
\hline Variables & $\mathbf{R}$ & Valor $\mathbf{F}$ & Valor & Valor t & Valor $\mathbf{p}$ \\
\hline Niveles V.B12 - tiempos IBP y diálisis & 0.15 & 0.392 & & & 0.678 \\
\hline Constante & & & 682.031 & 9.817 & 0.000 \\
\hline Coeficiente X1 & & & -11.731 & -0.817 & 0.555 \\
\hline Coeficiente X2 & & & 10.209 & 0.885 & 0.383 \\
\hline VCM - tiempos IBP y diálisis & 0.129 & 0.289 & & & 0.751 \\
\hline Constante & & & 92.715 & 48.456 & 0.000 \\
\hline Coeficiente X1 & & & -0.229 & -0.423 & 0.675 \\
\hline Coeficiente X2 & & & -0.046 & -0.143 & 0.887 \\
\hline $\mathrm{Hb}$ - Tiempos IBP y diálisis & 0.377 & 2.814 & & & 0.074 \\
\hline Constante & & & 11.071 & 30.172 & 0.000 \\
\hline Coeficiente X1 & & & 0.137 & 1.323 & 0.195 \\
\hline Coeficiente X2 & & & 0.027 & 0.441 & 0.662 \\
\hline
\end{tabular}




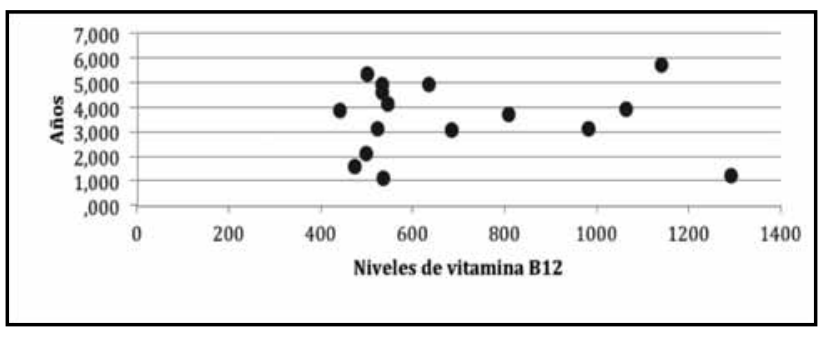

Figura 4. Relación entre niveles de vitamina B12 y uso de IBP entre uno y cinco años.

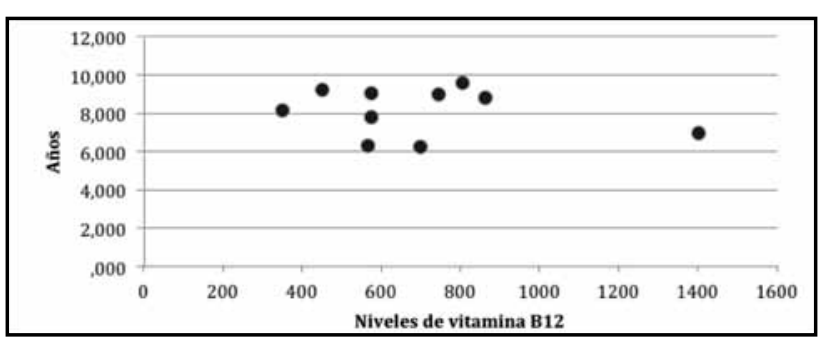

Figura 5. Relación entre niveles de vitamina B12 y uso de IBP por seis ó mas años.

\section{Discusión}

El término vitamina B12 hace referencia a los diferentes tipos de cobalaminas. La hidroxicobalamina y la cianocobalamina son formas no fisiológicas, que al ser consumidas se transforman en el organismo a metilcobalamina y 5 , desoxiadenosilcobalamina que son las formas fisiológicamente activas o coenzimas de la vitamina B12 (6).

Los alimentos de origen animal como la carne, huevos y leche son las principales fuentes en la dieta de vitamina B12. Una dieta occidental promedio contiene entre 5 y 15 microgramos por día, mucho más de los requerimientos necesarios que son aproximadamente 2 microgramos por día. El organismo tiene mecanismos muy eficientes de almacenamiento para esta vitamina, por tanto se requieren de dos a cinco años de bajo aporte nutricional para desarrollar una deficiencia clínica incluso teniendo una severa malabsorción (6).

El aparato digestivo posee un efectivo sistema para la absorción de la vitamina B12, en el cual normalmente intervienen tres tipos de proteínas: la haptocorrina, el factor intrínseco y la transcobalamina. El primer paso para la absorción de la cobalamina es su separación de las proteínas aportadas en los alimentos, esto ocurre por medio de la digestión péptica producto del ácido gástrico. Una vez liberadas, las cobalaminas y sus análogos se unen a las cobalofilinas (haptocorrinas o proteínas R) secretadas en la saliva creando una unión que resiste el pH gástrico. En el duodeno, la haptocorrina es hidrolizada por las enzimas pancreáticas y la cobalamina se une al factor intrínseco que fue secretado en el estómago y la protege de las enzimas proteolíticas; este complejo es absorbido en el íleon por el receptor de cubilina. Una vez dentro de la célula entérica el factor intrínseco es degradado y la cobalamina se libera para unirse a la transcobalamina II que es su principal transportador a los tejidos donde es metabólicamente activa. La transcobalamina I actúa como almacén temporal por su larga vida media y la transcobalamina III se encarga de transportar la cobalamina al hígado donde es almacenada (7).

La vitamina B12 es indispensable para el ser humano, $\mathrm{y}$ su deficiencia produce manifestaciones clínicas importantes. Esto se debe a que existen dos reacciones enzimáticas que requieren a la vitamina B12 como cofactor esencial.

La primera enzima es la metionina sintasa metilcobalamina dependiente, ésta se encarga de catalizar la metilación de homocisteína a metionina y también de la reducción de desoxi-uridinmonofosfato (dUMP) a desoxitimidil-monofosfato (dTMP). La implicación de su ausencia es que la célula puede producir ARN pero no ADN, presentándose un incremento en el ARN. Al tener una incapacidad de producir ADN de una forma apropiada la célula se queda estancada en la fase de síntesis (S) de la duplicación, no puede dividirse pero tiene un proceso normal de síntesis de ARN y proteínas, por lo tanto aumenta de volumen y se observa con núcleos grandes e inmaduros con cromatina laxa (6).

La incapacidad de producir nuevos eritrocitos adecuadamente y el estancamiento en una fase de crecimiento de la mitosis produce anemia megaloblástica, la más frecuente manifestación clínica de la deficiencia de vitamina B12. Esta disfunción en la mitosis no sólo afecta a los eritrocitos sino también a todas las demás líneas hematopoyéticas, de ahí que leucopenia y trombocitopenia pueden encontrarse acompañando la anemia megaloblástica. La segunda enzima es la metilmalonilCoA mutasa adenosil-cobalamina dependiente que se encarga de la isomerización del metilmalonilCoA a succinilCoA. Es importante para la reutilización mitocondrial en el ciclo de Krebs del propionilCoA producto de la oxidación de ácidos grasos de cadena impar. Éste déficit metabólico produce un estado de acidosis metabólica encontrándose un aumento del ácido metilmalónico en sangre y orina, elevación del ácido propiónico y en consecuencia cantidades elevadas de ácido acético en la orina.

La deficiencia en este proceso de isomerización ha sido asociada con desmielinización específica de la médula espinal (cordones dorsales y laterales de la médula espinal y la corteza cerebral) que ocurre en la deficiencia de vitamina B12 por el compromiso que produce en las membranas celulares. Dicha desmielinización se asocia con deficiencia de metionina, precursor de la $\mathrm{S}$ adenosil metionina necesaria para algunas reacciones esenciales en el mantenimiento de la mielina (8).

La deficiencia de vitamina B12 puede ocurrir por:

- Malabsorción de la vitamina, en la anemia perniciosa, gastrectomía o bypass gástrico, malabsorción de cobalamina anclada a proteínas, enfermedad o resección ileal, insuficiencia pancreática, malabsorción inducida por medicamentos (colchicina, neomicina, acido p-aminosalicílico, IBPs) y la ausencia congénita o disfunción del factor intrínseco (1). 
- Competencia biológica por la cobalamina dietaria que se da en síndromes de sobrecrecimiento bacteriano y en la infestación por tenias.

- Insuficiencia en la dieta más frecuente en los vegetarianos estrictos.

- Imposibilidad en su uso causada por la deficiencia congénita de la transcobalamina II. (1)

Los inhibidores de la bomba de protones (IBPs) son un grupo de medicamentos de gran uso clínico. Su función es la inactivación de la $\mathrm{H}+\mathrm{K}+$ trifosfatasa de adenosina gástrica, enzima que se encarga de la producción de ácido clorhídrico en las células parietales localizadas en las glándulas oxínticas en el epitelio gástrico. Todos los IBP se unen a la cisteína 813 de la subunidad alfa de la bomba de protones; se diferencian en que algunos se unen a otras cisteínas adicionalmente en la misma subunidad (9).

Los IBP dependen de la concentración ácida del medio estomacal para ejercer su función, pues en un medio ácido deben ionizarse, activándose bajo la forma de sulfenamidas lo que les permite unirse a las bombas de protones del canalículo secretor de la célula parietal mediante un enlace covalente. Por este efecto antisecretor del ácido gástrico estos medicamentos son de elección para patologías como la enfermedad úlcero-péptica, (10).

Los IBP son considerados medicamentos relativamente seguros, pero se ha reconocido que pueden tener un efecto negativo en la absorción de algunas vitaminas y minerales. Para la absorción efectiva de la vitamina B12 es necesario un medio ácido que cumpla la función proteolítica que separa dicha vitamina de las proteínas de la dieta; el uso de IBPs y la disminución en la acidez del medio estomacal podría disminuir la vitamina B12 disponible para la absorción. Aunque existe un fundamento lógico para dicho problema hay controversia en si su uso a largo plazo puede dar lugar a la generación de niveles séricos insuficientes.

Al respecto en el estudio de Lam y col, la utilización a largo plazo (dos o mas años) de IBPs se asoció con alto riesgo de deficiencia de vitamina B12 (10). Una revisión sistemática reciente concluye que el uso crónico de IBPs es un factor de riesgo para el desarrollo de deficiencia de vitamina B12 (12). Otros estudios realizados por LOTUS y SOPRAN, durante 12 y 5 años respectivamente, no demostraron que la utilización de IBP generaran diferencias significativas en los niveles séricos de vitamina B12 entre los pacientes del grupo control y los tratados (13).

Los pacientes con ERC sin diálisis podrían ser especialmente vulnerables a desarrollar déficit de vitamina B12 por factores nutricionales. Esto ocurre puesto que a la mayoría de los pacientes con enfermedad renal en etapa predialítica se les prescribe una dieta hipoproteica con el propósito de disminuir la progresión del daño renal crónico. Para el grupo de pacientes con ERC estadio 5 en terapia hemodialítica es también común mantener un estricto control de la dieta limitando el consumo de fosfatos y algunos tipos de proteínas en la dieta, con el fin de evitar la aparición de hiperfos- fatemia, hiperparatiroidismo secundario y complicaciones cardiovasculares (14).

Es recomendado que al paciente con ERC se le suministren $2.4 \mu \mathrm{g}$ /día de vitamina B12 $(15,16)$ En Colombia, a los pacientes con ERC se les suministran suplementos vitamínicos pero ninguno cumple con el contenido, ni dosis internacionalmente recomendados para las vitaminas hidrosolubles y en muchos también se encuentran vitaminas liposolubles en rangos no adecuados. Adicionalmente también existe el problema de la adherencia terapéutica encontrándose que sólo $70 \%$ de los pacientes hacen uso satisfactorio de sus suplementos multivitamínicos, es por esto que se recomienda que la administración de dichos suplementos se haga después de la diálisis (2).

Es de importancia resaltar que en los pacientes con ERC se usan con alta frecuencia los IBP, esto ocurre debido a que en ellos la pirosis, gastritis erosiva, duodenitis y la hemorragia digestiva son complicaciones frecuentes de la toxicidad urémica (4).

En nuestro estudio encontramos que contrariamente a lo esperado, los niveles de vitamina B 12 en una población de pacientes en hemodiálisis con muy variados periodos de tiempo fueron detectados en valores normales. Aunque la prescripción de complejo B fue un hallazgo común en la población estudiada (10 pacientes lo recibían), el contenido de las tabletas estaba limitado a vitamina B1 (tiamina), vitamina B2 (ribloflavina), vitamina B6 (piridoxina), y niacinamida. La edad de los pacientes, tiempo en diálisis, tiempo de consumo de IBPs, ni dosis de IBPs demostraron diferencias significativas en los resultados de los niveles de vitamina B12.

Una limitante de nuestro estudio puede ser el número de pacientes estudiados, puesto que se trata de una población pequeña, planteándose la posibilidad de desarrollar estudios multicéntricos con mayor número de pacientes, y en distintas regiones del país y con diversidad en los patrones culturales y nutricionales.

\section{Conclusiones}

Los niveles de vitamina B12 no se afectan por el consumo de IBP en pacientes con ERC en terapia de hemodiálisis. La determinación de sus niveles séricos podría restringirse a pacientes en hemodiálisis con otros factores de riesgo para generar su déficit.

\section{Conflicto de interés}

Los autores declaran que no tener conflictos de interés.

\section{Referencias}

1. Stabler S P. Vitamin B12 deficiency. N Engl J Med 2013; 368: 149-160.

2. Restrepo VCA, Varela MME, Valencia OHF. Estudio comparativo de los suplementos vitamínicos comercializados en Colombia y los requerimientos ideales en pacientes con enfermedad renal crónica en terapia dialítica. MÉD UIS 2010; 23: 189-97.

3. de-la-Coba C, Argüelles-Arias F, Martín-de-Argila C, Judez J, Linares A, Ortega-Alonso A et al. Proton-pump inhibitors adverse effects: a review of the evidence and position statement by the Sociedad Española de Patología Digestiva. Rev Esp Enferm Dig 2016; 108: 2 07-224.

4. Abu Farsakh NA, Roweily E, Rababaa M, Butchoun R. Brief report: evaluation of the upper gastrointestinal tract in uraemic patients undergoing haemodialysis. Nephrol Dial Transplant. 1996; 11: 847-850. 
5. Snow CF. Laboratory diagnosis of vitamin B12 and folate deficiency: a guide for the primary care physician. Arch Intern Med 1999; 159: 1289-1298.

6. Forrellat BM; Gómis HI; Gautier H. Vitamina B12: metabolismo y aspectos clínicos de su deficiencia. Rev Cubana Hematol Inmunol Hemoter 1999; 15: 159-174.

7. Rodríguez G P. Acido fólico y vitamina B12 en la nutrición humana. Revista Cubana Aliment Nutr 1998; 12: 107-119.

8. Leishear K, Boudreau RM, Studenski SA, Ferrucci L, Rosano C, de Rekeneire N, et al. Relationship between vitamin B12 and sensory and motor peripheral nerve function in older adults. J Am Geriatr Soc 2012; 60: 1057-1063

9. Oscanoa E TJ. Seguridad de los Inhibidores de la Bomba de Protones. Rev Gastroenterol Perú 2011; 31: 49-55.

10. García BA, Patrón RO, Sáez LM. Efectos a largo plazo de los inhibidores de bomba de protones. RAPD 2012; 5: 36-41.

11. Lam JR, Schneider JL, Zhao W, Zhao W, Corley D. Proton pump inhibitor and histamine 2 receptor antagonist use and vitamin B-12 deficiency. JAMA 2013; 310: 2435-2442
12. Jung SB, Nagaraja V, Kapur A, Eslick GD. Association between vitamin B12 deficiency and long-term use of acid-lowering agents: a systematic review and meta-analysis. Intern Med J 2015; 45: 409-16.

13. Attwood SE, Ell C, Galmiche JP, Fiocca R, Hatlebakk JG, Hasselgren B et al. Long-term safety of proton pump inhibitor therapy assessed under controlled randomised clinical trial conditions: data from the SOPRAN and LOTUS studies Aliment Pharmacol Ther 2015; 41: 1162-1174.

14. Nieto I, Mahecha P. Asociación entre hiperfosfatemia y mortalidad en los pacientes con insuficiencia renal crónica terminal en terapia de reemplazo de la función renal con diálisis peritoneal o hemodiálisis: Un estudio de la Unidad Renal de Villavicencio 1999 2003. Acta Méd Colomb 2005; 30: 14-18.

15. Heinz J, Domrose U, Westphal S, Luley C, Neumann K H, Dierkes J. Washout of water soluble vitamins and of homocysteine during haemodialysis: effect of high flux and low flux dialyser membranes. Nephrology 2008; 13: 384-9.

16.Descombes E, Hank A B, Fellay G. Water soluble vitamins in chronic hemodialysis patients and need for supplementation. Kidney Int 1993; 43: 1319-28. 